\title{
Hearing impairment in children following bacterial meningitis; assessed with brainstem auditory evoked response (BSAER)
}

\author{
Jayasinghe $\mathbf{S S}^{1,2}$, Pathirana $\mathrm{KD}^{2}$, Vimalasena GTHIP ${ }^{1}$, Tharanga $\mathbf{D}^{1}$ \\ ${ }^{\prime}$ Department of Pharmacology, Faculty of Medicine, University of Ruhuna, Galle, Sri Lanka \\ ${ }^{2}$ Clinical Neuroscience Center, Department of Medicine, Faculty of Medicine, Galle, Sri Lanka
}

Correspondence: Dr. S.S. Jayasinghe (sudheerasj@yahoo.com)

Source of funding: This study was supported by Wellcome Trust and NHMRC International Collaborative Research Grant (GR071669MA).

\begin{abstract}
Background: Bacterial meningitis is one of the most serious infections occurring in children. Sensorineural hearing loss is the commonest complication of bacterial meningitis. We aimed to assess the level of hearing in children following bacterial meningitis.

Materials and Methods: Retrospective study was conducted. The data were traced from April 2007 through August 2010 with the existing reports kept in the Clinical Neuroscience Center of the children following bacterial meningitis. Brainstem auditory evoked response (BSAER) was used to assess the level of hearing. The stimulation intensities of $90 \mathrm{~dB}, 60 \mathrm{~dB}$ and $30 \mathrm{~dB}$ were given to each ear separately. The occurrence of wave V was considered as effective conduction of stimulus to the auditory cortex.

Results: There were 30 (17 males) children following bacterial meningitis. All were below the age of five years. Eight children had meningitis during their neonatal period and five children 1-2 months of age, respectively. Seven required Intensive Care and four were ventilated. Sixteen (53.3\%) children had normal hearing. BSEAR of eight $(27 \%)$ children did not show wave $V$ in one of the sides to the stimulation intensity of $30 \mathrm{~dB}$. Three $(10 \%)$ did not have wave $\mathrm{V}$ in both sides to the stimulation intensity of $30 \mathrm{~dB}$. Four (13\%) had unilateral hearing loss to the stimulation intensity of $60 \mathrm{~dB}$. Two $(6 \%)$ children did not have wave V bilaterally to the stimulation intensity of $90 \mathrm{~dB}$.

Conclusion: Out of the children referred for BSEAR after meningitis, $47 \%$ had post meningitic hearing impairment. It is important to do BSEAR of all the children following bacterial meningitis to identify hearing impairment without delay.
\end{abstract}

Keywords: Evoked potentials, Meningitis

\section{Introduction}

Bacterial meningitis is one of the most serious infections occurring in children. This infection is associated with a high rate of acute complications and risk of long term morbidity. Sensorineural hearing loss (SHL) is the most common sequelae of bacterial meningitis (1). Usually, Sensorineural hearing loss presents at the time of initial presentation (1). Approximately $10 \%$ of survivors are affected $(2,3)$. It is recommended that all children with bacterial meningitis should undergo proper audiologic assessment before or soon after discharging from the hospital (1).

Subjective methods such as pure tone audiometry and visual reinforcement audiometry require the cooperation of the patient. Therefore these tests may not be possible with children. Brain stem auditory evoked response (BSAER) is an electro- 
physiological, non-invasive test which provides an objective measurement of hearing threshold. To some extent, the test can also differentiate the site of the lesion (4).

Delay in detecting hearing impairment after meningitis may lead to speech and learning difficulties, poor social interaction and psychological development in the later life. This may lead to misdiagnosis as mental retardation, autism or schizophrenia of childhood (1).

In Sri Lanka epidemiological studies are scant to find out the prevalence and consequences of bacterial meningitis. We aimed to asses the level of hearing after bacterial meningitis in children who were referred to the Clinical Neuroscience Center.

\section{Methods}

A study was conducted with the approval of the Ethical Review Committee, Faculty of Medicine, University of Ruhuna. The data were traced from April 2007 to August 2010 with the existing reports kept in the Clinical Neuroscience Center, Department of Medicine, Faculty of Medicine, University of Ruhuna, Galle.

A computer based Neuropack S1 EMG / EP measuring system MEB-9400 (Nihon Kohden) had been used for the BSAER. A piece of cotton moistened with alcohol was used to clean the patient's skin where the electrodes were attached and dry gauze was used to remove any moisture and gel from the skin. Recording electrodes (active and reference) and grounding electrode were attached on the patient's head with Elefix paste (Figure 1). Cooperative children kept on a chair relaxed with eyes closed. To avoid EMG of the neck, they were was asked not to move their head. Otherwise children were sedated with chloral hydrate $30 \mathrm{mg} / \mathrm{kg}$ body weight (5). The headphones were kept on the head. The hi-cut and the low-cut filters were set to $3 \mathrm{kHz}$ and $100 \mathrm{~Hz}$, respectively. The skin electrode contact impedance was kept below $5 \mathrm{k} \Omega$. The stimulation intensity was $90 \mathrm{~dB}, 60 \mathrm{~dB}$ and $30 \mathrm{~dB}$. The stimulus was given to each ear separately in descending order. Three thousand stimulations were averaged. The occurrence of wave $\mathrm{V}$ was considered as effective conduction of stimulus to the auditory cortex (6).

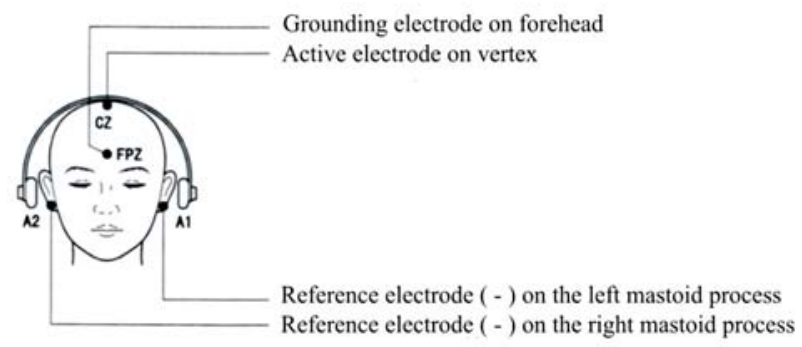

Figure 1: Electrode placement in BSEAR

\section{Results}

There were 30 ( 17 males) children who were referred to the Clinical Neuroscience Center, for hearing assessment following bacterial meningitis over the study period. All the patients were below the age of five years. Eight patients had meningitis during their neonatal period. Three got meningitis between one to two months of their lives. Seven out of 30 patients required Intensive Care and four of them were intubated and ventilated.

Figure 2 shows the presenting complaints at the time of presentation to the hospital. Fever was present in almost $70 \%$ of these patients.

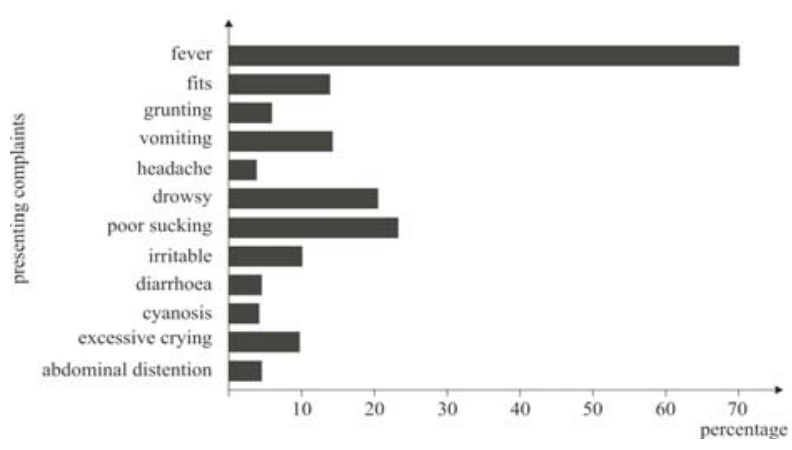

Figure 2: Presenting complaints at the time of admission to the hospital

Twenty-six patients had undergone lumbar puncture (LP) and 21 of them were diagnosed to have meningitis based on microscopy and biochemistry of CSF. Although LP of five patients came negative, the treating clinician had made the diagnosis of bacterial meningitis based on the clinical picture. Organisms were identified by culture in 1 of the 21 probable bacterial meningitis cases. Table 1 shows the laboratory results of the patients. Twelve patients had undergone US scan of the brain at the time of presenting to the Clinical Neuroscience Center, but none of them showed abnormalities. Computed 
Tomography (CT) scan of the brain had been done in four patients and three of them had subdural emphyma. All patients received antibiotics with the mean 1.4 (range 1-8) days after hospital admission. Eleven patients had received dexamethasone therapy but this information was not available in 19 patients.

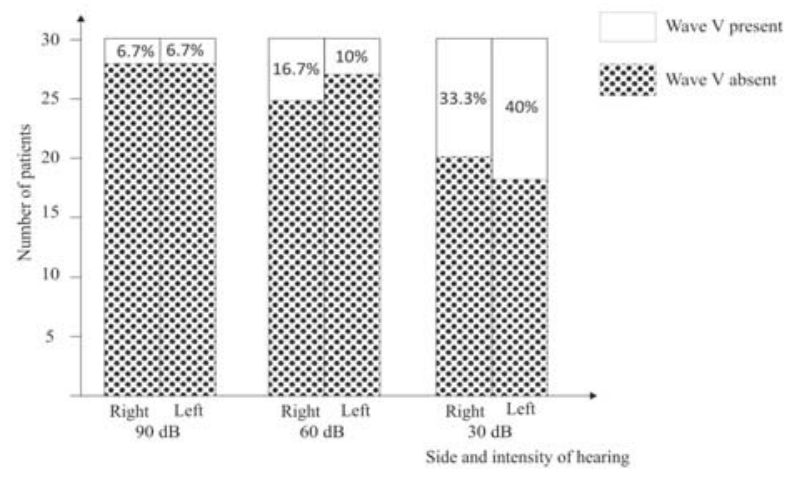

Figure 3: The level of hearing following bacterial meningitis following bacterial meningitis. BSEAR of eight $(26.6 \%)$ children did not show wave $\mathrm{V}$ in one of the sides to the stimulation intensity of $30 \mathrm{~dB}$, whereas three $(10 \%)$ children did not have wave $\mathrm{V}$ in both sides to the stimulation intensity of $30 \mathrm{~dB}$. Four $(13.3 \%)$ children had unilateral hearing loss to the stimulation intensity of $60 \mathrm{~dB}$. Two $(6 \%)$ children did not have wave $\mathrm{V}$ bilaterally even to $90 \mathrm{~dB}$ (Table 2). Among the seven patients who received intensive care, four patients had normal hearing. Two patients did not have wave $\mathrm{V}$ to $30 \mathrm{~dB}$ bilaterally (mild bilateral sensorineural hearing loss) and one patient did not have wave $\mathrm{V}$ even to $90 \mathrm{~dB}$ of stimulation intensity (severe sensorineural hearing loss). Three out of four patients who were ventilated had normal hearing and one patient had severe sensorineural hearing loss.

Sixteen $(53.3 \%)$ children had normal hearing

Table 1: Laboratory results of the patients

\begin{tabular}{llll}
\hline Interpretation & CSF full report & $\begin{array}{l}\text { CSF culture } \\
\text { ABST }\end{array}$ & $\begin{array}{l}\text { Blood Culture } \\
\text { ABST }\end{array}$ \\
\hline Positive & 21 & 1 & 2 \\
Negative & 5 & 22 & 20 \\
Contaminated & 0 & 1 & 2 \\
Data not available & 4 & 6 & 6 \\
\hline
\end{tabular}

CSF- cerebrospinal fluid, ABST- antibiotic sensitivity test

Table 2: Level of hearing impairment following bacterial meningitis

\begin{tabular}{|c|c|c|}
\hline Level of hearing & Number of children & Interpretation \\
\hline $90 \mathrm{~dB}, 60 \mathrm{~dB}$ and $30 \mathrm{~dB}$ & 16 & Normal \\
\hline \multicolumn{3}{|c|}{$90 \mathrm{~dB}$ and $60 \mathrm{~dB}$ (absent wave $\mathrm{V}$ to $30 \mathrm{~dB}$ ) } \\
\hline Unilateral & 5 & Unilateral mild hearing loss \\
\hline Bilateral & 3 & Bilateral mild hearing loss \\
\hline \multicolumn{3}{|c|}{$90 \mathrm{~dB}$ (absent wave V to $30 \mathrm{~dB}$ and $60 \mathrm{~dB}$ ) } \\
\hline Unilateral & $4 *$ & $\begin{array}{l}\text { Unilateral moderate to severe } \\
\text { hearing loss }\end{array}$ \\
\hline Bilateral & 0 & - \\
\hline \multicolumn{3}{|c|}{ None (absent wave V to $30 \mathrm{~dB}, 60 \mathrm{~dB}$ and $90 \mathrm{~dB}$ ) } \\
\hline Unilateral & 0 & Bilateral severe hearing loss \\
\hline Bilateral & 2 & - \\
\hline
\end{tabular}

*These patients had wave V on other side only up to $60 \mathrm{~dB}$ 


\section{Discussion}

Forty six percent of children who were referred for hearing assessment to our center had post meningitis hearing impairment to varying degree. This may be an overestimation as all meningitis patients were not referred for BSAER and clinicians may have decided to send only those with severe illness or suspected hearing impairment clinically due to lack of resources. Hence the results of this study may not represent all the children following meningitis.

As routine screening for hearing at birth is not feasible in Sri Lanka at present, at least all the high risk groups, especially those who had meningitis or ICU care need to be screened. A prospective study may give additional details pertaining to the actual prevalence of hearing loss after meningitis and risk factors of developing hearing impairment.

Richardson M P and Reid A (1997) assessed hearing of 110 children following meningitis (meningococcal 92, pneumococcal 18). All cases of hearing loss were apparent at the time of the first assessment within six hours of diagnosis. Three children $(2.4 \%)$ had permanent SHL (95\% CI 0.5 to 6.9). Thirteen children (10.5\%) had reversible hearing loss of whom nine had an impairment that resolved within 48 hours of diagnosis. The cochlea was identified as the site of the lesion in both the permanent sensorineural and reversible hearing impairments. Hearing loss was more common in children who had been ill for more than 24 hours (relative risk 2.72; 95\% CI 0.9 to 8) (9). Inpatient screening avoids delays in the diagnosis of post meningitis hearing loss. This is important because late diagnosis may prevent optimal audiologic rehabilitation. Cochlear implantation, for example, can partially restore hearing in post meningitis deafness, but the procedure may be ineffective if not performed promptly $(7,8)$. It may not be possible to do BSEAR of all the children following bacterial meningitis due to limited resources. It is clear that inpatient screening is very important for early detection followed by BSEAR to confirm the diagnosis and for the subsequent intervention.

\section{Acknowledgments}

The authors like to thank all the pediatricians who referred patients to the Clinical Neuroscience Center, Galle. We wish to thank Mrs. Kumuduni de Silva, Mrs. Sunethra Weerarathna and the demonstrators of the Department of Medicine, Faculty of Medicine, University of Ruhuna for their technical support and data entry.

\section{References}

1. Kliegman RM, Jenson HB, Behrman RE, Jenson HB, Stanton BF. Nelson text book of Paediatrics, 2008; $18^{\text {th }}$ edition, 602:2512-2523.

2. Fortnum HM. Hearing impairment after bacterial meningitis: a review. Arch Dis Child, 1992; 67: 1128-1133.

3. Baraff LJ, Lee SI, Schriger DL. Outcomes of bacterial meningitis in children: a meta-analysis. Pediatr Infect Dis J, 1993; 12: 389-394.

4. Richardson MP, Williamson TJ, Reid A, Tarlow MJ, Rudd PT. Outoacoustic emissions as a screening test for hearing impairment in children recovering from acute bacterial meningitis. Paediatrics, 1998; 102: 1364-1368.

5. British Medical Association. Hypnotics. British National Formulary, 2002; 4: 169-170.

6. Aminof MJ. Brainstem auditory evoked potentials. Electrodiagnosis in Clinical Neurology, 2005; 5: 489-524.

7. Gibson WP, Brown C, Everingham C, Herridge S, Rennie M, Steinberg T. Necessity of early diagnosis and assessment of post meningitis children in view of cochlear implantation. Ann Otol Rhinol Laryngol, 1995; 104: 208210 .

8. Dodds A, Tyszkiewicz E, Ramsden R. Cochlear implantation after bacterial meningitis: the dangers delay. Arch Dis Child, 1997; 76: 139-140.

9. Richardson MP, Reid A, Tarlow MJ, Rudd PT. Hearing loss during bacterial meningitis. Arch Dis Child, 1997; 76(4): 386. 